\title{
ANÁLISIS DE LA SITUACIÓN ACTUAL DEL TURISMO DE SOL Y PLAYA EN EL CARIBE: UN ESTUDIO DE CASO
}

\author{
Francisco Orgaz Agüera \\ Área de Turismo y Empresas \\ Universidad de UTESA, República Dominicana ${ }^{1}$ \\ franorgaz@hotmail.com \\ Francisco José Ortega Fraile ${ }^{2}$ \\ Departamento de Economía Financiera y Dirección de Operaciones \\ Universidad de Sevilla \\ fjof83@hotmail.com
}

\begin{abstract}
Resumen
En los últimos años las islas han ido desarrollando un cambio significativo en su modelo económico, mejorando y segmentando su oferta turística. De esta forma, además del clásico turismo de sol y playa, se ha incentivado la oferta cultural, gastronómica o ecológica. El destino objeto de nuestro estudio, República Dominicana, se consolida como uno de los principales destinos de esta tipología turística en la región del Caribe. En este artículo presentamos los resultados de una investigación, basada en entrevistas y observación de campo, con la finalidad de analizar la situación actual del turismo de sol y playa en República Dominicana, para lo cual se ha contado con la colaboración de diferentes actores implicados en el desarrollo del turismo en el país. Los principales resultados de esta investigación se muestran a través de una matriz DAFO (debilidades, amenazas, fortalezas y oportunidades).
\end{abstract}

\section{Palabras clave}

turismo de sol y playa, oferta complementaria, polos turísticos, islas, República Dominicana

\begin{abstract}
In recent years the islands have developed a significant change in its economic model, improving and segmenting its tourism offer. Thus, besides the classic sun and beach tourism, the cultural, gastronomic and ecological offer has been encouraged. The target destination of our study, Dominican Republic, has become one of the major tourist destinations of this type in the Caribbean region. In this paper, we present the results of an investigation, based on interviews and field observation, in order to analyze the current situation of tourism of sun and beach in Dominican Republic, for which it has enjoyed the cooperation of different actors involved in tourism development in the country. The main results of this research can be highlighted through a SWOT matrix (weaknesses, threats, strengths and opportunities).
\end{abstract}

\section{Keywords}

sun and beach tourism, complementary offer, tourist poles, islands, Dominican Republic

\section{INTRODUCCIÓN}

El desarrollo de la actividad turística genera oportunidades para la modernización socioeconómica y cultural de un destino (Castellanos Verdugo y Orgaz Agüera, 2013), y, en este sentido, las islas están transformando sus modelos económicos a través de las exportaciones de productos del sector primario y el desarrollo de la construcción. Según Correia et al. (2008), las islas son el segundo destino más importante para las vacaciones del turista, solo por detrás de las ciudades históricas, y esto está provocando que estos destinos estén siendo estudiados, en temática turística, por numerosos investigadores (Bryan, 2001; Craigwell, 2007; Roberts y LewisCameron, 2010; López-Guzmán et al., 2011).

En este sentido, siguiendo a Cameron y Gatewood (2008), el buen clima existente en estos destinos y el atractivo que genera en los turistas la separación física de estas zonas de los continentes, provocan la elección de estos lugares por parte de los turistas para sus vacaciones, si bien a estos atractivos se le deben añadir otros como los recursos naturales y culturales existentes en estas zonas, con la finalidad de ofrecer respuestas a los viajeros exigentes que buscan nuevas formas de turismo. Así, siguiendo a Vanegas y Croes (2003), el desarrollo del turismo en las islas requiere de nuevas estrategias que den respuestas a las exigencias de la demanda, las cuáles deben realizarse a través de estudios empíricos, con la finalidad de dar a conocer el mercado del destino y formular nuevas acciones que mejoren la competitividad del destino a nivel internacional (López-Guzmán et al.,

\footnotetext{
${ }^{1}$ Doctorando en Turismo

2 Doctorando en Turismo
} 
2011), si bien, según Castellanos Verdugo y Orgaz Agüera (2013), se debe tener presente que el grado de satisfacción de la demanda dependerá de las características físicas, humanas y organizativas de los servicios y/o productos turísticos que se oferten en un destino específico.

Centrándonos en República Dominicana, destino objeto de análisis de esta investigación, el turismo de sol y playa se configura como la principal tipología turística en el país (Agosín et al., 2009), aunque, el desarrollo del turismo de masas en el país ha provocado numerosos impactos negativos en el medio natural y, por lo tanto, se necesita desarrollar nuevas formas de turismo más sostenibles (Orgaz Agüera, 2013). En este sentido, el objetivo principal de esta investigación es analizar la situación actual del turismo de sol y playa en República Dominicana, con la finalidad de evaluar la situación de esta tipología de turismo en el país caribeño, a través de un estudio cualitativo, basado en la observación participante y en las entrevistas a los agentes que gestionan las zonas naturales en el norte del país, complementando así la información obtenida a partir de fuentes secundarias. También, este artículo pretende reforzar la literatura existente sobre la actividad turística en este país caribeño, debido a la importancia que este sector ha adquirido en las islas como a los nuevos cambios del perfil de turista, que busca actividades complementarias al clásico turismo de sol y playa en el destino. Para reflejar mejor los resultados realizaremos un análisis a través de las debilidades, amenazas, fortalezas y oportunidades (DAFO) del desarrollo del turismo de sol y playa en República Dominicana.

Para lograr estos objetivos, este artículo se estructura, tras esta introducción en un segundo apartado donde se desarrolla una revisión de la literatura sobre turismo de sol y playa, siguiéndole un tercer apartado donde se describirá el área geográfica. Posteriormente, en un cuarto apartado se describe la metodología de esta investigación y en el quinto apartado se muestran los principales resultados de esta investigación mediante un DAFO. Finalmente, se presentan las conclusiones más relevantes de esta investigación.

\section{REVISIÓN DE LA LITERATURA}

La situación del turismo de sol y playa en la actualidad comienza a ser una realidad diferente según nos muestran diversos autores como Bigné Alcañiz et al (2008), García Sánchez y Alburquerque García (2003) o Rivas (2004). Así pues, aunque sigue siendo un importante factor motivador a la hora de elegir destino vacacional, después de las ciudades históricas, se comienza a replantear un nuevo paradigma en torno a esta tipología turística, y es que cada vez más existe un rechazo al turismo masivo, buscando experiencias individualizadas y variadas (Aguilo et al, 2005, Rivas, 2004). De forma que el modelo tradicional debe ir adoptando nuevos caminos, como nos indica Ivars (2003) y Spanks (2003), es decir, diversificar la oferta complementaria y mejorar la calidad de los servicios turísticos.

El desarrollo del turismo en el siglo XXI requiere de nuevas líneas estratégicas que den respuesta a una demanda cada vez más exigente, y que huye cada vez más de la masificación turística, repartiendo sus vacaciones a lo largo de todo el año (Mazars Turismo, 2002). En esta línea, según el estudio realizado por García Sánchez y Alburquerque García (2003), se observa que las nuevas tendencias apuntan a segmentos del mercado muy similares tanto en el turismo cultural y como en el de sol y playa. Y esto es debido principalmente al fraccionamiento de las vacaciones, a la evolución de la ocupación durante los meses más castigados por la estacionalidad, a un mayor nivel cultural y al surgimiento de nuevas inquietudes y preferencias por parte del turista.

Por otra parte, se ha de tener en cuenta que la construcción de grandes complejos en los destinos turísticos ha provocado la pérdida de vegetación, la alteración de la fauna y la contaminación aérea, generando un turismo de masas que ha contribuido y contribuye en la actualidad al agravamiento de las zonas geográficas afectadas (Orgaz Agüera, 2014), aspecto que no deja de ser más que el resultado de un crecimiento turístico descontrolado del pasado, tal como como nos indica Knowles y Curtis (1999). En este sentido, debe ser planteado un nuevo paradigma de turismo de sol y playa dentro de la actividad turística del futuro, pues el nuevo turista reclama un turismo que contemple tres aspectos básicos, tales como la calidad de los servicios, infraestructuras e instalaciones, una responsabilidad medioambiental en la actividad desarrollada y un compromiso de integración y respeto por población local donde tiene lugar la actividad turística (García Sánchez y Alburquerque García, 2003). De esta forma, y tal como se señalaba al comienzo de esta revisión bibliográfica, la calidad y la diversificación son claves en la nueva andadura del turismo de sol y playa. Un cliente satisfecho generará calidad en la oferta turística, pero una buena diversificación de productos y servicios es muy importante para mejorar la competitividad, de manera que se incrementará el valor de los servicios y productos turísticos, dando lugar a la generación de nuevos mercados. Así pues, el turismo cultural se presenta como una importante alternativa complementaria a la tradicional actividad turística centrada exclusivamente en sol y playa. Por tanto, y según Zaratiegui (2002), las ciudades precisan de reclamos relevantes para captar turistas de todas clases, y es por ello por lo que se ha de enriquecer la oferta turística de sol y playa con elementos culturales, que impregnarán de un mayor valor no sólo a la ciudad, sino a los servicios y productos turísticos que en las mismas se oferten. En esta misma línea, Manning et al (2002), Fraile (2002) y Zacarías et al (2011) nos indica que la existencia de elementos culturales en los destinos de sol y playa constituyen un importante atractivo a la hora de la elección de los destinos por parte de los turistas, de forma que el patrimonio, los diferentes tipos de eventos culturales, el folklore o los festivales entre otra oferta complementaria serán elementos diferenciadores que contribuyan a un mejor posicionamiento y venta del destino. En definitiva, el turista busca, cada vez más, vivir experiencias de primera mano, así como el contacto y el conocimiento de la población autóctona del destino elegido.

Hay un elemento transversal a todo lo anterior, que es la ruptura de la estacionalidad como elemento clave en los destinos de sol y playa, un hecho que según García Sánchez y Alburquerque García (2003), se viene 
produciendo en los últimos años, pues se vienen registrando mayores crecimiento de ocupación en los meses que quedan fuera de la temporada veraniega, y que puede ser motivado por la tendencia al fraccionamiento de las vacaciones así como por la huida del cliente de las saturaciones que se producen en los destinos durante la época estival. La desestacionalización se plantea, por tanto, como un elemento crucial para imprimir valor añadido a los destinos de sol y playa, generando importantes ventajas para todos los agentes implicados de forma directa o indirecta en el sector turístico, pues contribuye a que la mano de obra se vuelva más cualificada, incidiendo muy positivamente en la productividad y economía de las empresas implicadas.

Podemos concluir este apartado con tres principales ideas, y es el que el turismo de sol y playa persigue no sólo el descanso, sino también el esparcimiento, de ahí la importancia de la oferta complementaria que diversifique el modelo tradicional centrado solamente en el sol y la playa (Martínez, 2001 y Rivas 2004). Por otra parte, podemos entender que esta diversificación viene de mano de los cambios producidos en la demanda, pues nos encontramos con un consumidor con más facilidad de acceso a la información, más exigente y con las ideas mucho más claras en su elección vacacional. De esta manera, todo se traduce en mayores exigencias de calidad, y por tanto, en una intensificación de la competencia (Bigné Alcañiz et al, 2008). Finalmente, y tal como nos indica Zacarias et al (2011), la conservación y la gestión en los destinos de sol y playa adquieren un papel muy importante, de cara a mitigar los efectos negativos que esta tipología turística pueda provocar, por lo debe ser una responsabilidad común a todos los agentes implicados para garantizar la perdurabilidad del destino.

\section{DESCRIPCIÓN DEL ÁREA GEOGRÁFICA}

El turismo en República Dominicana se caracteriza por un turista que visita el país para alojarse en hoteles-resort "todo incluido", sin la necesidad de salir al exterior, debido a que en el mismo resort encuentra todas las comodidades que precisa para satisfacer las necesidades de su viaje (Moreno Gil et al., 2002), como son la playa, actividades de ocio y deportivas, discotecas, bares, restaurantes, casino $u$ otras actividades complementarias. Según Villarreal y Van Der Horst (2008), la actividad turística se configura como uno de los principales sectores económicos en República Dominicana.

En este sentido, la Asociación de Hoteles y Turismo de la República Dominicana (ASONAHORES ${ }^{3}$ ), principal organismo que proyecta datos mensuales sobre la situación actual del turismo en el país, además de informar diariamente sobre las diversas noticias que hay en torno al sector turístico tanto a nivel nacional como internacional, establece que el país caribeño recibió durante el año 2013 la cifra total de 4.689 .770 visitantes (turistas y nacionales no residentes), lo que supone un 2,79\% más que el año anterior, donde se registraron, según esta misma institución, un total de 4.562 .606 visitantes, incluyéndose aquí tanto los visitantes extranjeros como los dominicanos no residentes en el país.

También, y según datos de esta misma organización sobre el número total de turistas que llegaron al país (tabla 1), podemos observar que Punta Cana es el principal destino turístico del país, recibiendo su aeropuerto el $63,59 \%$ de visitantes. Posteriormente, le sigue Santo Domingo (capital del país) y Puerto Plata.

Tabla 1: Principales aeropuertos dominicanos en llegadas de turistas internacionales durante el año 2013

\begin{tabular}{|c|c|}
\hline AEROPUERTOS & CIFRAS DE TURISTAS \\
\hline Punta Cana & 2.584 .871 \\
\hline Las Américas (Santo Domingo) & 800.218 \\
\hline Puerto Plata & 353.857 \\
\hline Cibao & 168.446 \\
\hline La Romana & 91.481 \\
\hline El Catey (Samaná) & 54,126 \\
\hline La Isabela (El Higüero) & 11.755 \\
\hline
\end{tabular}

Fuente: Elaboración propia a partir de datos de ASONAHORES (2013)

Teniendo en cuenta lo anterior, y según Agosín et al., (2009), en República Dominicana se puede observar seis polos turísticos diferenciados (figura 1), que pasamos a desarrollar a continuación.

\footnotetext{
${ }^{3}$ www.asonahores.com
} 
Figura 1: Polos turísticos de República Dominicana.

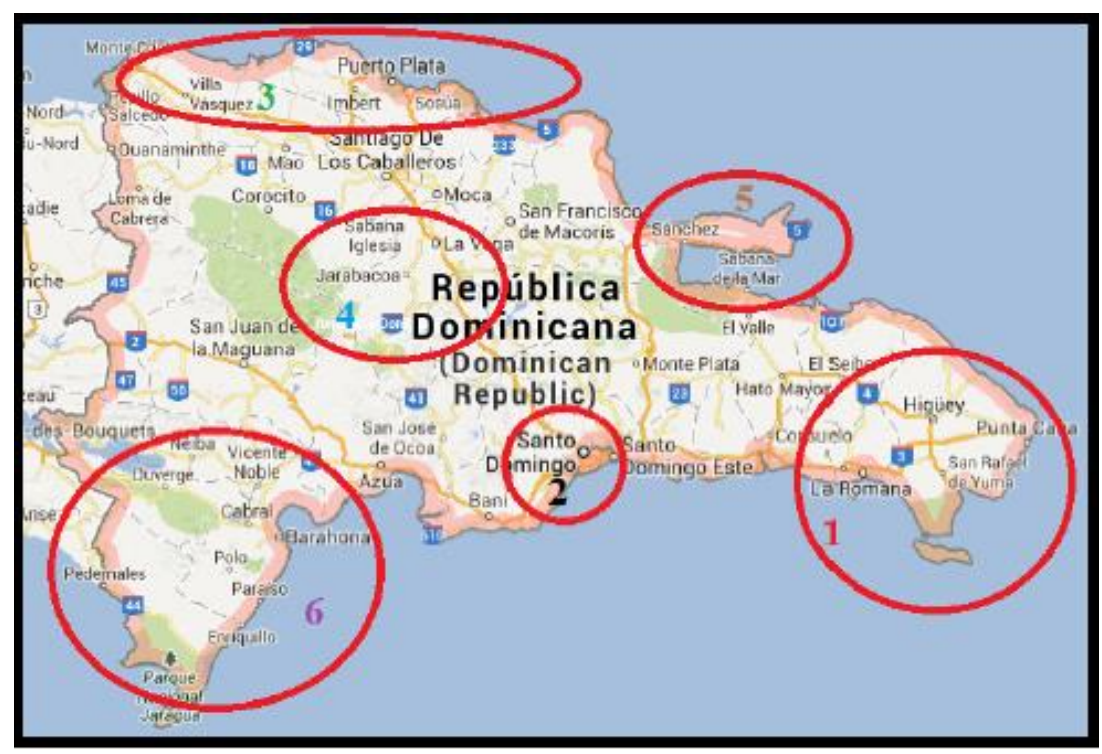

Fuente: Elaboración propia a través de Google Maps.

En este sentido, en la región este del país (número 1, figura 1) se divisa el principal desarrollo turístico de sol y playa del país, con destinos consolidados a nivel mundial, como son Punta Cana, La Romana, Bayahibe y Bávaro. Además, ahí se encuentra el área protegida más visitada del país, Parque Nacional del Este, beneficiándose de las excursiones que hacen los turistas de los hoteles-resort a la Isla Saona, comercializándose esta área desde finales del siglo pasado (Girault, 1998).

Santo Domingo (número 2, figura 1), capital de República Dominicana, se caracteriza por viajeros de negocios, lo que ha significado también un descenso del turismo de sol y playa en la zona de Boca Chica, principal playa de la capital (Agosín et al., 2009), aunque, en esta zona también destaca las playas de Juan Dolio. Así, actualmente se configura como el segundo destino del país tras la región este.

En la región norte y noroeste destaca el desarrollo turístico de sol y playa y deportivo en Puerto Plata (número 3 , figura 1), en ocasiones beneficiando algunas otras provincias cercanas, menos desarrolladas turísticamente pero con gran potencial, como es el caso de Montecristi (Agosín et al., 2009). Esta región es el segundo polo turístico de sol y playa, tras Punta Cana, debido a como hemos dicho anteriormente, que Santo Domingo ha descendido el turismo de sol y playa, y se está consolidando como un destino de negocios.

En el centro del país se encuentran las ciudades de Constanza y Jarabacoa (número 4, figura 1), donde se ubican los principales destinos de montaña de República Dominicana (Agosín et al., 2009), destacando la zona más alta del país y de todo El Caribe: Pico Duarte.

En la región noreste, en concreto en la península de Samaná (número 5, figura 1), se está produciendo un rápido crecimiento del turismo inmobiliario, además del propio turismo de sol y playa (Agosín et al., 2009). También, y según Agosín et al., (2009), destaca el turismo de cruceros, consolidado desde la temporada 2005-2006. Aunque, en la época de febrero-marzo, esta región destaca por prácticas ecoturísticas relacionadas con el avistamiento de la ballena jorobada.

Por último, en la costa suroeste (número 6, figura 1) existe un enorme potencial para el desarrollo del turismo ecológico, pero no está lo suficientemente desarrollado, si bien, empiezan a incorporarse pequeñas empresas locales que ofertan excursiones por las áreas protegidas (Agosín et al., 2009). Esta zona además es una de las ricas en avifauna, lo que la hace un área con importantes potenciales para actividades como la ornitología.

\section{METODOLOGÍA}

La metodología utilizada para el desarrollo de esta investigación ha consistido en fuentes primarias y secundarias. En primer lugar, las fuentes secundarias han consistido en la revisión de la literatura a través de artículos científicos de otros investigadores y académicos, y datos y cifras elaboradas por instituciones dominicanas, para realizar la primera parte de este trabajo, correspondiente al marco teórico de esta investigación y la descripción del área geográfica.

Por otro lado, las fuentes primarias han consistido en métodos cualitativos, a través de la entrevista y la observación participante. En este sentido, la entrevista consiste en un acto de comunicación oral que se establece entre dos o más personas, con el fin de obtener una información (Malhotra Naresh, 2004). Estas entrevistas se establecieron entre los autores de este trabajo y profesionales que trabajan en el sector turístico de República Dominicana. También se entrevistaron a algunas personas pertenecientes a las comunidades locales, que tienen una relación muy estrecha con el turismo. Por último, también fueron entrevistados políticos pertenecientes al Ministerio de Medio Ambiente y Recursos Naturales del país. El número total de personas 
entrevistadas fue de 24. Por su parte, la observación participante, ha consistido en visitar todos los polos turísticos anteriormente mencionados, para conocer de primera mano cuales son las principales potencialidades de estas zonas, así como, para realizar un análisis del desarrollo turístico de sol y playa más real. En este sentido, hemos utilizado una metodología de carácter cualitativa debido a que la consideramos la más adecuada para la realización de esta investigación, y en concordancia con las herramientas utilizadas en la misma.

Para ver con más claridad los resultados obtenidos en la investigación, se presentan a través de un análisis DAFO (debilidades, amenazas, fortalezas y oportunidades). Con esta matriz se evaluarán los puntos fuertes y débiles del sector junto con las amenazas y oportunidades externas al mismo, habiendo sido utilizada esta herramienta por diversos autores en sus estudios turísticos (Sariisik et al., 2011; Romero Macías et al., 2011; Reihanian et al., 2012; Zhang, 2012).

\section{RESULTADOS DE LA INVESTIGACIÓN Y DISCUSIÓN}

Los resultados de esta investigación se muestran en base a una matriz DAFO. Así, para ver con mayor claridad los resultados obtenidos, mostraremos las siguientes tablas sobre las debilidades, amenazas, fortalezas y oportunidades encontradas en este estudio.

En este sentido, en primer lugar presentaremos una serie debilidades (tabla 2), que afectan de forma negativa en el desarrollo del turismo de sol y playa de República Dominicana.

Tabla 2: Debilidades del turismo de sol y playa en República Dominicana

\begin{tabular}{|c|c|}
\hline $\begin{array}{l}\text { DEBILIDADES } \\
\text { (Weaknesses) }\end{array}$ & $\begin{array}{l}\text { 1. No hay mucha variedad de actividades complementarias al turismo de sol y playa. } \\
\text { 2. El transporte público no está bien organizado y en muchas ocasiones pretenden } \\
\text { aprovecharse de los turistas. } \\
\text { 3. Inadecuados modelos de marketing de algunos polos turísticos. } \\
\text { 4. Baja seguridad de los turistas a la hora de salir de los resorts. } \\
\text { 5. Mala percepción del turista sobre las zonas aledañas a los hoteles por temas } \\
\text { relacionados con las condiciones de vida de los residentes. } \\
\text { 6. Deficiencia en infraestructura básica para viajar fuera del hotel-resort. } \\
\text { 7. Baja capacitación de los empleados de los complejos hoteleros. }\end{array}$ \\
\hline
\end{tabular}

Fuente: Elaboración propia.

Como se observa en la tabla 2, encontramos que República Dominicana no dispone de una gran variedad de ofertas complementarias fuera del hotel-resort, teniendo en cuenta su gran potencial para desarrollar otras tipologías turísticas (Castellanos Verdugo y Orgaz Agüera, 2013). En este sentido, solamente la región este con el Parque Nacional del Este (Isla Saona), la región norte con el Parque Nacional Isabel de Torres y el Monumento Natural Saltos de la Damajagua, y Samaná con el Parque Nacional Los Haitises, tienen ofertas complementarias al turismo de sol y playa consolidadas. Así, existen muchos otros recursos culturales, monumentales y naturales con un gran potencial para configurar ofertas complementarias, pero no existen una oferta con tales recursos, como ya han destacado algunos autores (Castellanos Verdugo y Orgaz Agüera, 2013; Orgaz Agüera y Lopez-Gúzman, 2013).

Por otro lado, no existen adecuados modelos de marketing en algunos polos turísticos, lo que genera una débil promoción a nivel nacional e internacional, generalmente cuando se pretende publicitar la oferta complementaria de la zona, que hoy en día es muy importante para que el turista se decida por un lugar u otro. Incluso, algunas zonas tienen deficiencia tanto en su infraestructura como en las vías de comunicación, siendo el caso por ejemplo de la región sur o del noroeste del país (Montecristi).

En cuanto a la seguridad, República Dominicana en general es un país no muy seguro para los turistas, debido al alto nivel de delincuencia y pobreza que azota al país, y que en muchos casos provocan robos a los turistas. En este mismo sentido, el transporte público es también un factor importante a tener en cuenta, debido a que es abundante y tiene un precio asequible en el país, pero en ocasiones se aprovechan de los turistas cobrándole más caro (Orgaz Agüera y López-Guzmán, 2013).

Otra debilidad es la calidad del servicio, debido a que muchos empleados tienen nivel de formación muy bajo, y por tanto, su trato con el turista en ocasiones no tiene la calidad esperada, aunque, por el contrario, los dominicanos son personas abiertas y muy agradables, lo que a veces, genera que el turista reciba un buen trato. Así, aunque el empleado dominicano es una persona que trasmite una gran simpatía para el turista, la baja formación, en ocasiones, hace que su trato hacia el turista no sea el esperado por este, y generalmente, esto se produce por la falta de capacitaciones y formación de los empleados. Esto solo ocurre en los puestos de trabajo más bajos (limpiadoras, seguridad, camarero, etc.) y no en los directores generales o de departamento y recepción. Aunque, esta debilidad es corregida por muchos hoteles-resort, a través de políticas de responsabilidad social corporativa que se realizan a partir de acciones de formación y capacitación a los empleados.

Por su parte, el desarrollo de la actividad de sol y playa en el país puede suponer una serie de amenazas (tabla 3) que pueden llegar a afectar al desarrollo de dicha actividad en República Dominicana. 
Tabla 3: Amenazas del turismo de sol y playa en República Dominicana

\begin{tabular}{|c|c|c|}
\hline $\begin{array}{l}\text { AMENAZAS } \\
\text { (Threats) }\end{array}$ & $\begin{array}{l}2 . \\
3 .\end{array}$ & $\begin{array}{l}\text { La construcción de grandes complejos hoteleros provoca perdida de vegetación } \\
\text { y alteración de la fauna en las playas. } \\
\text { La actividad turística de masas provoca contaminación ambiental. } \\
\text { El turismo de sol y playa es la principal tipología turística en el país, faltando } \\
\text { ofertas complementarias a este turismo (falta de diversificación de oferta). } \\
\text { Ausencia de planes de desarrollo turísticos en algunos polos turístico, lo que ha } \\
\text { generado diversos problemas en la zona. } \\
\text { Precios de los billetes aéreos muy altos, dependiendo la región dominicana a la } \\
\text { que se desee llegar. } \\
\text { Existencia de destinos competitivos muy cerca a República Dominicana }\end{array}$ \\
\hline
\end{tabular}

Fuente: Elaboración propia

Por tanto, según la tabla 3, la construcción de los grandes complejos hoteleros puede provocar tanto la perdida de la vegetación como la alteración de la fauna, además de contaminar el área (Agosín et al., 2009), como ya ha ocurrido en algunas regiones del país (Orgaz Agüera, 2013), aunque según Wearing y Neil (2000), en cualquier región del planeta pueden aparecer estos impactos negativos referentes a la actividad ecoturística. Esto también se agrava posteriormente con las llegadas de turistas, debido a que el turismo de sol y playa es una tipología de masas que causa, en ocasiones, contaminación en áreas de playas.

El turismo de sol y playa también se configura como el principal tipo de turismo de República Dominicana, aunque, el hotel resort debe ser complementado. No solo atractivos tales como parques de diversiones, sitios naturales, eventos, excursiones y reliquias históricas que induzcan al turista a visitarlos, sino también facilidades de alojamiento y amenidades de otros tipos como los ecolodges, hoteles de montaña y proyectos de turismo comunitario. Así, según ASONAHORES (2014), el destino se torna más completo y, por ende, el hotel del "todo incluido" tiene mayor valor agregado.

También, cabe resaltar que no existen planes de desarrollo turístico adecuado en algunas regiones donde se practica la actividad turística de masas, lo que ha provocado que el turismo de sol y playa no se desarrolle de forma adecuada y bajo los principios de la sostenibilidad.

Por último, los precios de los billetes aéreos son muy altos, dependiendo la región dominicana a la que se desee llegar, siendo más bajos si se contrata con agencia chárter en "todo incluido". Esto, junto a la existencia de destinos competitivos muy cerca a República Dominicana, está generando que el turismo haya frenado su subida en el último año.

Por su parte, la actividad turística de sol y playa en República Dominicana dispone de numerosas fortalezas (tabla 4) para su desarrollo.

Tabla 4: Fortalezas del turismo de sol y playa en República Dominicana

\begin{tabular}{|l|ll|}
\hline & 1. & Destino consolidado en turismo de sol y playa "todo incluido". \\
FORTALEZAS & Existencia de grandes cadenas hoteleras internacionales y nacionales \\
(Strengths) & en los diferentes polos turísticos.
\end{tabular}

Fuente: Elaboración propia

Como se puede observar en la tabla 4, República Dominicana cuenta con numerosas fortalezas, siendo la principal, que el país está consolidado a nivel internacional en turismo de sol y playa, sobre todo, la región este que destaca con la llegada del $63,59 \%$ de los turistas. Esto se debe a la existencia de grandes cadenas hoteleras internacionales y nacionales distribuidas por las playas del país, y la, también, existencia de aeropuertos internacionales, destacando el de Punta Cana. En todo caso, las cadenas hoteleras se encuentran ubicadas cerca de los principales aeropuertos, conectados a través de buenas vías de comunicaciones.

La consolidación de República Dominicana como destino de sol y playa a nivel internacional se debe a la buena imagen internacional de calidad y el paisaje de sus costas, generada por la vegetación, la arena fina y blanca, así como la calidad de sus aguas, sobre todo, las de las Costa del Mar Caribe.

Por último, encontramos las oportunidades (tabla 5), que deben ser aprovechadas para mejorar el turismo de sol y playa en República Dominicana. 
Tabla 5: Oportunidades del turismo de sol y playa en República Dominicana

\begin{tabular}{|l|ll|}
\hline & 1. & Grandes recursos potenciales para mejorar la oferta complementaria. \\
OPORTUNIDADES & 2. Alto grado de motivación por parte de los empresarios locales en el país. \\
(Opportunities) & 3. Nuevas vías de ingresos para la provincia a través del turismo de masas. \\
& 4. Creación de nuevas empresas y de empleo. \\
\hline
\end{tabular}

Fuente: Elaboración propia

Debemos destacar, según la tabla 5, que en República Dominicana existe un gran potencial natural, monumental y cultural en el país, para crear nuevas ofertas complementarias, que pueden comercializarse en los hotelesresort, y que tienen como objetivo mejorar la oferta complementaria dirigida a los turistas, y por otro lado, mejorar el desarrollo socioeconómico en el país, a través de la creación de empleo y creación de nuevas empresas. Sobre todo, la naturaleza dominicana tiene grandes potencialidades, debido a que el país dispone de 123 áreas protegidas (Reyna Alcántara y Polonia Martínez, 2012), para poner en marcha actividades ecoturísticas (Castellanos Verdugo y Orgaz Agüera, 2013). En este sentido, existe interés por parte de las administraciones públicas (Ministerio de Turismo y Ministerio de Medio Ambiente y Recursos Naturales) para el desarrollo de actividades turísticas en polos turísticos con potencial para el turismo de sol y playa, implementando en ellas nuevas ofertas complementarias.

Existe un alto grado de motivación por parte de los empresarios locales (Castellanos Verdugo y Orgaz Agüera, 2013) para poner en marcha productos y/o servicios turísticos cerca de los hoteles-resort, lo que puede generar nuevas vías de ingreso para República Dominicana, a través de un aumento de la calidad de vida de la población local que se fomenta con la creación de nuevos empleos y empresas. Esto, se puede fortalecer con las cooperaciones que existen entre los diferentes sectores y empresas, y con las ONG internacionales que operan en el país.

\section{CONCLUSIONES}

Tras el trabajo de campo e investigación planteado en el presente documento, podemos decir que República Dominicana presenta importantes potencialidades en lo que a la explotación turística se refiere. Si bien, su principal actividad turística se ha centrado tradicionalmente en el turismo masivo de sol y playa, hemos comprobado que esta tipología no sólo debe cambiar por exigencia de la demanda, sino que este giro se hace de obligado cumplimiento para garantizar y preservar la actividad turística, el medioambiente, el patrimonio y la identidad dominicana.

República Dominicana reúne una serie de condiciones ambientales y patrimoniales que convierten al país en un centro turístico muy competitivo, pero aún por desarrollar y explotar. En este sentido, se ha de huir del turismo masificado que generan los grandes resorts alojados en el país, para ofertar un turismo comprometido con la población local, sus espacios naturales y su historia. Para ello, se han de mejorar aspectos como la seguridad, el transporte público y las condiciones de vida de los residentes, generando de esta forma una mejor imagen del destino, y que inciten al turista a conocer el país, traspasando las "fronteras" de los resorts hoteleros y sus paradisiacas playas caribeñas.

Cambiar el rumbo del turismo que se produce en República Dominicana es cuestión de iniciar adecuadas campañas de marketing, capacitar a los empleados de las empresas turísticas, así como ayudar a la población local a generar riqueza a partir de los recursos que en el país se encuentran, complementando y diversificando la oferta turística. Igualmente, hay una serie de acciones que deben de venir de la mano de las autoridades competentes, acciones que deben ir encaminadas a la consecución de planes de desarrollo en algunos polos turísticos, así como una regulación de los precios aéreos, de cara a tratar justamente a todas las regiones dominicanas.

En definitiva, no se plantea ni mucho menos romper con todo lo anterior, sino un cambio de concepto aprovechando todo lo bueno que República Dominicana ya tiene, es decir, su consolidación como destino de sol y playa, la presencia de importantes empresas hoteleras y la existencia de aeropuertos internacionales debe ser el germen de la creación de una oferta complementaria, sostenible y comprometida con la población autóctona.

\section{BIBLIOGRAFÍA}

AGOSÍN, M., MANZANO, O., RODRÍGUEZ-BALZA, R. y STEIN, E. (2009): La ruta hacia el crecimiento sostenible en la República Dominicana: Fiscalidad, competitividad, institucionalidad y electricidad. Santo Domingo: Banco Interamericano de Desarrollo (BID).

AGUILÓ, E., ALEGRE, J. y SARD, M. (2005): "The persistence of the sun and sand tourism model", Tourism Management, 26, pp. 219-231.

ASONAHORES (2014): Estadísticas turísticas 2013 República Dominicana. Disponible en: http://www.asonahores.com/estad\%C3\%ADsticas/bolet\%C3\%ADn-mensual/2014/1/bolet\%C3\%ADnestad\%C3\%ADstico-diciembre-2013.aspx. Consultado: 21/03/2014. 
BIGNÉ ALCAÑIZ, E, SÁNCHEZ GARCÍA, I. y CURRÁS PÉREZ, R. (2007): "El papel de la imagen del destino en la valoración y comportamiento postcompra del turista de sol y playa". Papers de Turisme, 42, 57-73.

BRYAN, A. (2001): Caribbean tourism: Igniting the engines of sustainable growth. Florida: University of Miam.

CAMERON, C. M., y GATEWOOD, J. B. (2008): "Beyond sun, sand and sea: The emergent tourism programme in the Turks and Caicos Islands". Journal of Heritage Tourism, 3 (1), 55-73.

CASTELLANOS VERDUGO, M. y ORGAZ AGÜERA, F. (2013): "Potencialidades ecoturísticas de la República Dominicana". TURyDES, Revista Científica en Turismo y Desarrollo Local, 6 (14), 1-15.

CORREIA, A.; BUTLER, R. y OLIVEIRA, N. (2008): "First-time and repeat visitors to Cape Verde. The overall image". Tourism Economics, 14 (1), 185-204.

CRAIGWELL, R., (2007). Tourism competitiveness in small island developing states, Helsinki: World Institute for Development Economics Research (WIDER).

FRAILE GONZÁLEZ, F. (2002): El fenómeno turístico, I Congreso Internacional de Turismo Cultural. Www.turismo-cultural.org.

GARCÍA SÁNCHEZ, A. y ALBURQUERQUE GARCÍA, F. J. (2003): "El turismo cultural y el de sol y playa: ¿sustitutivos o complementarios? Cuadernos de Turismo, 11, 97-105.

GIRAULT, C. (1998): "El auge del Turismo en la República Dominicana: Implicación social y política”. Ciencia y sociedad, 23 (3), 417-426.

IVARS, J.A. (2003): "Regional development policies: an assessment of their evolution and effects on the Spanish tourist model", Tourism Management, 24, pp. 655-663.

KNOWLES, Y. y CURTIS, S. (1999): "The market viability of European mass tourist destinations. A poststagnation lifecycle analysis", International Journal of Tourism Research, 1, pp. 87-96.

LOPEZ-GUZMAN, T.; BORGES, O. y CASTILLO-CANALEJO, A. M., (2011): "Community based tourism in Cape Verde: A case study". Tourism and Hospitality Management, 17 (1), 34-44.

MALHOTRA NARESH, K. (2004): Introducción y primeras fases de la investigación de mercados. Mexico: Pearson Educación Prentice Hall.

MANNING, R., WANG, B., VALLIERE, W., LAWSON, S., y NEWMAN, P. (2002). "Research to estimate and manage carrying capacity of a tourist attraction: a study of Alcatraz Island". Journal of Sustainable Tourism, 10 (5), 388-403.

MARTÍNEZ, R. (2001): "Aproximación al estudio del sector turístico almeriense: análisis de la oferta y de la demanda en temporada alta", Cuadernos de Turismo, 7, pp. 81-91.

MAZARS TURISMO (2002): Aproximación al turismo residencial. Ed. FITUR 2002.

MORENO GIL, S.; CELIS SOSA D. F. y AGUILAR QUINTANA, T. (2002): "Análisis de la satisfacción del turista de paquetes turísticos respecto a las actividades de ocio en el destino: El caso de República Dominicana". Cuadernos de Turismo, 9, 67-84.

ORGAZ AGÜERA, F. (2013): "Análisis del impacto ambiental en República Dominicana a través de la ley sobre medio ambiente y recursos naturales". DELOS, Revista Desarrollo Local Sostenible, 6 (17), 1-10.

ORGAZ AGÜERA, F. y LÓPEZ-GUZMÁN, T. (2013): "El ecoturismo como actividad complementaria al turismo de sol y playa. Un análisis de caso en Puerto Plata, República Dominicana". En: Perna, F.; Gouveia, P.; Serra, F.; Santos, J. y Águas, P. (2013). Economia, Ambiente e Sustentabilidade no Turismo. Faro: Universidade do Algarve, pp. 5-15.

ORGAZ AGÜERA, F. (2014): "El Ecoturismo en los Humedales: Análisis de las Potencialidades de República Dominicana". Revista Rosa dos Ventos, 6 (1), 4-18.

REIHANIAN, A.; BINTI MAHMOOD, N. Z.; KAHROM, K. y WAN HIN, T. (2012): "Sustainable tourism development strategy by SWOT analysis: Boujagh National Park, Iran". Tourism Management Perspectives, 4, 223-228.

REYNA ALCANTARA, E. y POLONIA MARTINEZ, A. (2012): ATLAS de biodiversidad y recursos naturales de República Dominicana. Santo Domingo: Ministerio de Medio Ambiente y Recursos Naturales.

RIVAS, J. (2004): Estructura y Economía del Mercado Turístico, Septem Ediciones, España.

ROBERTS, S. y LEWIS-CAMERON, A. (2010): "Small island developing status: Signs and prosperts". En LEWISCAMERON, A. (coord.) (2010). Marketing island destinations: Concepts and cases. Oxford: Elsevier, 110. 
ROMERO MACÍAS, E., ROMERO MACÍAS, V. y VARGAS SÁNCHEZ, A. (2011): "El turismo rural y el Parque Natural Sierra de Aracena y Picos de Aroche de la provincia de Huelva (España): Una visión de futuro". PASOS, Revista de Turismo y Patrimonio Cultural, 9 (4), 503-517.

SPANKS (2003): "Tourism in Spain”, Nosk Handelskammer, Octubre.

ZACARÍAS, D. A., WILLIAMS, A. T. y NEWTON, A. (2011): "Recreation carrying capacityestimations to support beach management at Praia de Faro, Portugal". Applied Geography, 31, 1075-1081.

ZARATIEGUI, A. (2002): La ciudad turística: Los retos de una gestión innovadora. I Congreso Internacional sobre el Desarrollo Turístico Integral de Ciudades Monumentales. Granada, Febrero 2002.

ZHANG, X. (2012): "Research on the Development Strategies of Rural Tourism in Suzhou Based on SWOT Analysis". Energy Procedia, 16 (Part B), 1295-1299.

SARIISIK, M., TURKAY, O. y AKOVA, O. (2011): "How to manage yacht tourism in Turkey: A swot analysis and related strategies". Procedia, Social and Behavioral Sciences, 24, 1014-1025.

VANEGAS, M. y CROES, R. (2003): "Growth, development and tourism in a small economy: Evidence from Aruba". International Journal of Tourism Research, 5 (5), 315-330.

VILLAREAL, R. y VAN DER HORST, A. (2008): "Estrategia de competitividad turística de la República Dominicana". ARA, Journal of Tourism Research, 1 (1), 15-28.

WEARING, S., y NEIL, J. (2000): Ecoturismo. Impacto, tendencias y posibilidades. Madrid: Síntesis. 